\title{
Robust conditioned flavor preferences with a sensory preconditioning procedure
}

\author{
SANDRA A. LYN and ELIZABETH D. CAPALDI \\ University of Florida, Gainesville, Florida
}

\begin{abstract}
During sensory preconditioning, rats were given two distinct flavors (saccharin and coffee) in sequence with 0,9 , or $27 \mathrm{sec}$ between the two flavors. A control group received the flavors unpaired. In subsequent training, the second flavor preceded sucrose by $5 \mathrm{~min}$. Later, the subjects that had had 0 - or 9-sec delays between the two flavors showed a significant preference for the flavor not directly reinforced, whereas those that had had a 27 -sec delay showed only a marginally significant preference. Although taste aversions have been produced using these methods, this is the first demonstration of conditioned preferences for flavors not directly associated with the reinforcers. These results offer an alternative way to study flavor-flavor learning.
\end{abstract}

There are two known methods to produce conditioned flavor preferences. In the first, an arbitrary flavor, $x$, is mixed with an already preferred food or flavor. In the second, an arbitrary flavor, $x$, precedes a preferred food or flavor. In both methods, preference is customarily measured by giving a two-bottle preference test between the conditioned flavor, $x$, and another flavor, $y$, a flavor that was not paired with the preferred food or flavor. A conditioned preference is manifest by greater consumption of flavor $x$ than of flavor $y$.

In both of these methods the flavor is directly paired with the reinforcer. Animals can come to prefer the flavor because of the taste of the reinforcer (flavor-flavor learning) or because of its postingestive consequences (flavor-nutrient learning). One way to distinguish between these, it has been suggested (Capaldi \& Sheffer, 1992), is temporal delay. Capaldi and Sheffer found that a flavor preference can be conditioned by a caloric reinforcer, even when that reinforcer is delayed by $2 \mathrm{~h}$. There have been no studies varying the delay in flavorflavor preference conditioning. Lavin (1976) is often cited as having shown that flavor-flavor learning is not possible with a between-flavors delay greater than 9 sec. However, Lavin used aversive conditioning, not preference conditioning. In a cross-experiment comparison, Holman (1975) found that dextrose could reinforce flavor preferences with a delay of $30 \mathrm{~min}$, but saccharin could not. This is consistent with the hypothesis that flavor-nutrient learning is possible at a

This research was supported in part by Grant MH 39453 to the second author from the National Institute of Mental Health. Requests for reprints should be sent to E. D. Capaldi, Department of Psychology, University of Florida, Gainesville, FL 32611-2065. longer delay than is flavor-flavor learning. The purpose of the present experiment was to measure flavorflavor learning at various delays in the conditioned preference procedure.

Our procedures followed those of Lavin (1976), who gave saccharin followed by coffee in Phase 1 and poisoned rats for drinking coffee in Phase 2. Later he found an aversion to saccharin. Here, we gave saccharin followed by coffee in Phase 1 and followed coffee by sucrose in Phase 2 . We varied the delay between saccharin and coffee, using delays of 0,9 , and $27 \mathrm{sec}$, as did Lavin. If the saccharin-coffee pairings produce an association between saccharin and coffee, and if coffee-sucrose pairings produce a preference for coffee, this method may produce a conditioned preference for saccharin. Effects of varying the delay between saccharin and coffee will indicate how flavor-flavor learning varies with delays up to $27 \mathrm{sec}$.

Lavin (1976) referred to this method as sensory preconditioning. In sensory preconditioning, two stimuli are paired. The value of one of the stimuli is then changed by pairing it with an unconditioned stimulus (US), and a test phase measures whether that change in value transfers to the other stimulus. The textbook definition of sensory preconditioning specifies that the two stimuli should initially be neutral (e.g., Domjan, 1993), but Lavin's results show that this procedure also works when the stimuli initially produce affective reactions of their own. The sensory preconditioning procedure has previously not been used to produce conditioned flavor preferences. Some believe that conditioned aversions are stronger than conditioned preferences (e.g., Rozin, 1968; Rozin \& Kalat, 1971) and thus believe that conditioned preferences may not be strong enough to transfer to stimuli not directly associated with the 
reinforcer. It is also interesting in this regard that, heretofore, the majority of the sensory preconditioning demonstrations, beginning with Brogden (1939), have used aversive conditioning (e.g., Archer \& Sjoden, 1982; Barnet, Grahame, \& Miller, 1991; Lavin, 1976; Revusky, 1980). There have been demonstrations of sensory preconditioning using appetitive USs (Beninger, Hanson, \& Phillips, 1980; Rescorla, 1984), but these studies used audiovisual conditioned stimuli (CSs), not flavor CSs.

Fudim (1978) used flavor CSs in a study referred to as sensory preconditioning, but the procedure was not a sensory preconditioning one. Fudim gave rats two flavor CSs mixed with either salt or sugar. Later, when a sodium need was induced, the rats preferred the flavor that had been mixed with salt. Fudim's results showed that the rats had associated the flavor with salt (flavorflavor learning) and that inducement of a need for salt enhanced preference for a flavor associated with salt. That is, he modified the value of one of the stimuli-not by pairing it with a US, as in sensory preconditioning, but by a deprivation manipulation. Fedorchak and Bolles (1987) also found that deprivation of a commodity increased preference for flavors associated with that commodity. In their case, food deprivation increased preference for flavors associated with calories. In Fudim's as well as Fedorchak and Bolles's studies, preferences were measured for flavors that had been directly associated with the reinforcer.

One reason sensory preconditioning and flavor-flavor learning are confused is that both have been investigated recently by mixing flavors together and both have been interpreted in terms of within-compound associations. But if no conditioning follows the association of two flavors, the phenomenon cannot properly be termed sensory preconditioning. It is still left to be demonstrated whether sensory preconditioning is possible using flavor CSs and an appetitive US.

\section{METHOD}

\section{Subjects}

The subjects were 48 naive, male Sprague-Dawley rats from Harlan Co., Indianapolis, IN. At the start of the experiment they were between 73 and 75 days old. They were individually housed and tested in standard cages constructed of sheet-metal sides and wire-mesh bottoms. Lights were on at 6:00 a.m. and off at 6:00 p.m. All sessions were conducted at 9:00 a.m.

\section{Materials and Apparatus}

Solutions $(\mathrm{w} / \mathrm{v})$ were presented in $50-\mathrm{ml}$ Nalgene tubes topped with rubber stoppers fitted with metal spouts. The CS1 solution was $0.15 \%$ saccharin from the Sigma Chemical Co., and the CS2 solution was $1.25 \%$ Sanka naturally decaffeinated instant coffee. Lavin (1976) found that it made no difference whether coffee or vinegar was used as the CS2, and thus we did not counterbalance here. In training, a $20 \%$ sucrose solution was used as the reinforcer. All solutions were made with distilled water.

\section{Procedure}

The procedure followed that of Lavin (1976).

\section{Preliminary Switch Training}

Prior to the start of the experiment, the animals had ad-lib access to food and water. On Day 1, water bottles were removed from the rats' cages and for the next 5 days they had access to $20 \mathrm{ml}$ of water each day. Ad-lib food was available inside their cages throughout the experiment. On Day 6, the subjects began switch training. They were trained to consume $3 \mathrm{ml}$ of water from tubes placed to the left of the cage front. Upon complete consumption, the tubes were removed and $3 \mathrm{ml}$ of water was then made available to them in tubes to the right of the cage front. These were removed after depletion. There were three daily sessions of switch training, with a 1 -min intertrial interval (ITI) between each trial. This was the only source of water during this phase. After 5 days of switch training, the sensory preconditioning phase was implemented.

\section{Sensory Preconditioning Phase}

Three pairings of CS1 and CS2 were administered to the rats in the experimental groups each day for 10 days. The CS1 was put on the front left side of the cages and, after complete consumption, the tubes were removed and $3 \mathrm{ml}$ of CS2 was administered to the right. After complete consumption, the tubes were removed. This association of the CS1 and the CS2 constituted one trial. There were three such associations of the CS 1 and CS2 each day, with a 1-min ITI between each pairing. The interstimulus interval (ISI) was dependent on the group. There were four groups of 12 rats each. Group 0 had a 0 -sec delay between the CS1 and the CS2; thus, coffee (CS2) immediately followed saccharin (CS1). Groups 9 and 27 had the same conditions, except that their ISIs were 9 and $27 \mathrm{sec}$, respectively. The fourth group, the control group, had no association between the CS1 and the CS2. On odd days, the control group received CS1 followed immediately by CS1, and on even days they had CS2 followed immediately by CS2. During this phase, the CS1 and CS2 solutions were the only source of fluids for all the groups.

\section{Training}

All the groups were given $5 \mathrm{ml}$ of CS2 (coffee), followed $5 \mathrm{~min}$ later by $5 \mathrm{ml}$ of sucrose solution. It took approximately $3 \mathrm{~min}$ for each rat to consume $5 \mathrm{ml}$ of either the coffee or sucrose solutions. The solutions were presented on the right or left, according to an ABBA schedule. On the first day, CS2 was presented to the left and the sucrose solution to the right. On the next 2 days, the CS2 was presented to the right and the sucrose solution to the left, the next day was like the first, and then the schedule was repeated. Ten minutes after each session, the rats received $10 \mathrm{ml}$ of water. Training lasted for 10 days.

\section{Testing}

All the groups were given two-bottle choice tests between $40 \mathrm{ml}$ of CSI, saccharin, and $40 \mathrm{ml}$ of distilled water for $10 \mathrm{~min}$ a day for 2 days. On the first day the CSI was on the left, and water was on the right. The positions were reversed on the second day. The amount of solutions consumed for each subject was measured and recorded. There were no data collected until the test phase, because all solution amounts were fixed during the prior phases.

\section{RESULTS}

Figure 1 shows the mean consumption (in milliliters) of saccharin and water over both test days for each group. As can be seen in Figure 1, all the groups drank more saccharin solution than water; that is, they preferred the saccharin. The control group shows the size of the unconditioned preference for saccharin, and they showed the smallest preference. The preference was somewhat larger in Group 27, larger yet in Group 9, and largest in Group 0.

An analysis of variance on amount (milliliters) consumed included saccharin versus water (termed preference hereafter) and days as the within factors and groups as the between factor. The preference for saccharin over water was significant $[F(1,44)=134.69, p<.001]$ and varied significantly by group $[F(3,44)=4.83, p<.001]$. 


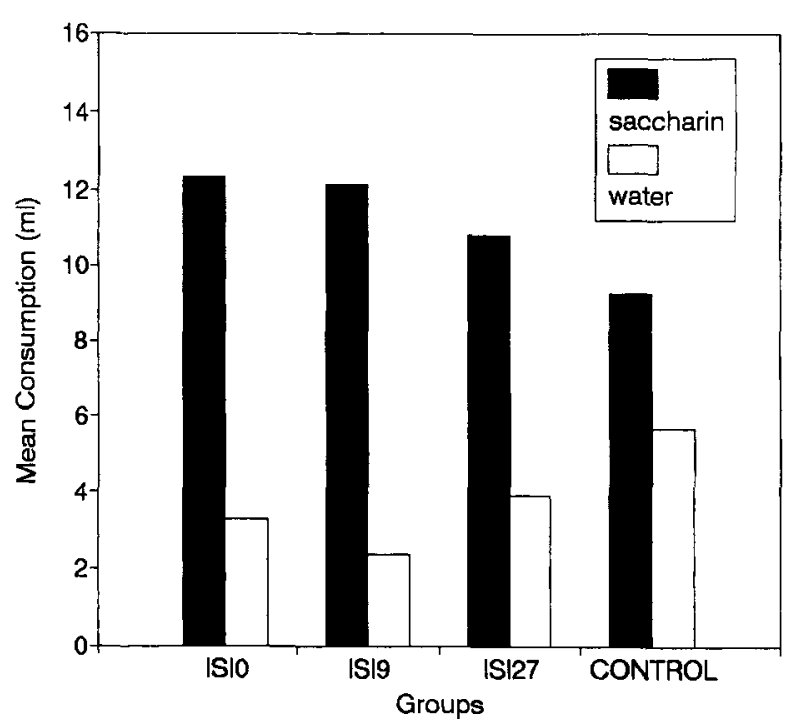

Figure 1. Mean consumption of saccharin and water for each group in test, with interstimulus intervals (ISIs) of 0, 9, and $27 \mathrm{sec}$.

Subsequent Newman-Keuls tests $(p<.05)$ showed that the preference for saccharin over water was not significant for the control group, but was significant for each of the other groups. Preference did not change significantly over the 2 days $[F(1,44)=2.17, p>.05]$ and days also did not affect group preference $[F(1,44)=0.50, p>$ $.05]$.

To see which of the experimental groups showed a preference larger than that of the control group, comparisons of each group's preference with that of the control were conducted. Group 0 (ISI 0 ) showed a preference that was significantly larger than that of the control group $[F(1,22)=9.38, p<.01]$, as did Group 9 (ISI $9 \mathrm{sec}$ ) $[F(1,22)=11.97, p<.01]$. Group 27 (ISI $27 \mathrm{sec}$ ), however, showed only a marginally significant larger preference than that of the control group $[F(1,22)=3.41$, $0.05<p<.10]$. All $F$ s refer to group $\times$ preference interactions.

\section{DISCUSSION}

The results challenge the view that conditioned preferences are weak (e.g., Rozin, 1968; Rozin \& Kalat, 1971). Pairing coffee with sucrose produced a preference for the saccharin associated with coffee. All the groups consumed equal amounts of coffee, saccharin, and sucrose in training; thus, preferences cannot be attributed to differential familiarity. The results show that conditioned flavor preferences are strong enough to transfer to a flavor not directly associated with the reinforcer.

Like Lavin (1976), we found that sensory preconditioning was effective when 0 or $9 \mathrm{sec}$ intervened between saccharin and coffee, but not when $27 \mathrm{sec}$ intervened between the two flavors. In contrast, flavor-nutrient associations can be made with an interval of $30 \mathrm{~min}$ between flavor and reinforcer (e.g., Capaldi, Campbell, Sheffer, \& Bradford, 1987). In the present study, conditioned flavor preferences were produced for the saccharin associated with coffee, when coffee preceded sucrose by $5 \mathrm{~min}$. Clearly, flavor-nutrient learning spans longer intervals than does flavor-flavor learning.

One speculation is that flavor-flavor learning involves associations between sensory aftereffects that are short term and hence cannot bridge a delay. The flavor of food involves both odor and taste, neither of which is long-lasting. In flavor-flavor learning, both the cue and the reinforcer are short term. In contrast, in flavor-nutrient learning, only the cue is short term, and reinforcing nutrients last a long time. Perhaps the long duration of nutrient digestion creates a stronger reinforcer, or stronger memory of reinforcer, one capable of bridging the delay.

Here, flavors were presented sequentially prior to pairing one of the flavors with an appetitive US. This sensory preconditioning procedure can be used to evaluate effects of variables on flavor-flavor learning separately from effects during flavor preference conditioning - something not possible in the usual conditioned preference design, in which flavors and reinforcer are presented mixed together.

\section{REFERENCES}

ARCHER, T., \& SJÖDÉN, P. O. (1982). Higher-order conditioning and sensory preconditioning of a taste aversion with an exteroceptive $\mathrm{CS}_{1}$. Quarterly Journal of Experimental Psychology: Comparative \& Physiological Psychology, 34, 1-17.

Barnet, R. C., Grahame, N. J., \& Miller, R. R. (1991). Comparing the magnitudes of second-order conditioning and sensory preconditioning effects. Bulletin of the Psychonomic Society, 29, 133-135.

Beninger, R. J., Hanson, D. R., \& Phillips, A. G. (1980). The effects of pipradrol on the acquisition of responding with conditioned reinforcement: A role for sensory preconditioning. Psychopharmacology, 69, 235-242.

BROGDEN, W. J. (1939). Sensory preconditioning. Journal of Experimental Psychology, 25, 323-332.

Capaldi, E. D., Campbell, D. H., Sheffer, J. D., \& Bradford, J. P. (1987). Conditioned flavor preferences based on delayed caloric consequences. Journal of Experimental Psychology: Animal Behavior Processes, 13, 150-155.

CAPAldi, E. D., \& ShefFer, J. D. (1992). Contrast and reinforcement in consumption. Learning \& Motivation, 23, 63-79.

DOMJAN, M. (1993). The principles of learning and behavior. California: Brooks/Cole.

FEDORCHAK, P. M., \& Bolles, R. C. (1987). Hunger enhances the expression of calorie- but not taste-mediated conditioned flavor preferences. Journal of Experimental Psychology: Animal Behavior Processes, 13, 73-79.

Fudim, O. K. (1978). Sensory preconditioning of flavors with a formalin-produced sodium need. Journal of Experimental Psychology: Animal Behavior Processes, 4, 276-285.

Holman, E. C. (1975). Immediate and delayed reinforcers for flavor preference in rats. Learning \& Motivation, 6, 91-100.

LAVIN, M. J. (1976). The establishment of flavor-flavor associations using a sensory preconditioning procedure. Learning \& Motivation, 7, 173-183.

Rescorla, R. A. (1984). Associations between Pavlovian CSs and context. Journal of Experimental Psychology: Animal Behavior Processes, 10, 195-204.

RevusKY, S. (1980). A sensory preconditioning effect after a single flavor-flavor pairing. Bulletin of the Psychonomic Society, 15, 83-86.

RozIN, P. (1968). Specific aversions and neophobia as a consequence of vitamin deficiency and/or poisoning in half-wild and domestic rats. Journal of Comparative \& Physiological Psychology, 66, 82-88.

Rozin, P., \& Kalat, J. W. (1971). Specific hungers and poisoning as adaptive specializations of learning. Psychological Review, $\mathbf{7 8 ,}$ 459-486.

(Manuscript received May 8, 1994; revision accepted for publication July 11, 1994.) 